\title{
Specific retention of the protostome-specific PsGEF may parallel with the evolution of mushroom bodies in insect and lophotrochozoan brains
}

\author{
Nozomu Higuchi, Keigo Kohno and Tatsuhiko Kadowaki*
}

Address: Graduate School of Bioagricultural Sciences, Nagoya University, Chikusa, Nagoya 464-8601, Japan

Email: Nozomu Higuchi - fabugroove_07@hotmail.co.jp ; Keigo Kohno - keigo0709@yahoo.co.jp;

Tatsuhiko Kadowaki* - emi@nuagr1.agr.nagoya-u.ac.jp

* Corresponding author

BMC Biology 2009, 7:21 doi:|0.1|86/|74|-7007-7-2|

This article is available from: http://www.biomedcentral.com/174I-7007/7/2I

(c) 2009 Higuchi et al.; licensee BioMed Central Ltd.

This is an open access article distributed under the terms of the Creative Commons Attribution License (http://creativecommons.org/licenses/by/2.0), which permits unrestricted use, distribution, and reproduction in any medium, provided the original work is properly cited.

\begin{abstract}
Background: Gene gain and subsequent retention or loss during evolution may be one of the underlying mechanisms involved in generating the diversity of metazoan nervous systems. However, the causal relationships acting therein have not been studied extensively.

Results: We identified the gene PsGEF (protostome-specific GEF), which is present in all the sequenced genomes of insects and limpet but absent in those of sea anemones, deuterostomes, and nematodes. In Drosophila melanogaster, PsGEF encodes a short version of a protein with the $\mathrm{C} 2$ and PDZ domains, as well as a long version with the C2, PDZ, and RhoGEF domains through alternative splicing. Intriguingly, the exons encoding the RhoGEF domain are specifically deleted in the Daphnia pulex genome, suggesting that Daphnia PsGEF contains only the C2 and PDZ domains. Thus, the distribution of PsGEF containing the $\mathrm{C} 2, \mathrm{PDZ}$, and RhoGEF domains among metazoans appears to coincide with the presence of mushroom bodies. Mushroom bodies are prominent neuropils involved in the processing of multiple sensory inputs as well as associative learning in the insect, platyhelminth, and annelid brains. In the adult Drosophila brain, PsGEF is expressed in mushroom bodies, antennal lobe, and optic lobe, where it is necessary for the correct axon branch formation of alpha/beta neurons in mushroom bodies. PsGEF genetically interacts with Racl but not other Rho family members, and the RhoGEF domain of PsGEF induces actin polymerization in the membrane, thus resulting in the membrane ruffling that is observed in cultured cells with activated forms of Rac.

Conclusion: The specific acquisition of PSGEF by the last common ancestor of protostomes followed by its retention or loss in specific animal species during evolution demonstrates that there are some structural and/or functional features common between insect and lophotrochozoan nervous systems (for example, mushroom bodies), which are absent in all deuterostomes and cnidarians. PsGEF is therefore one of genes associated with the diversity of metazoan nervous systems.
\end{abstract}




\section{Background}

A comparison of the genomes of five insects and five vertebrates revealed that there were approximately 1,000 genes present in all the insects but absent in the vertebrates. In contrast, there were approximately 5,000 genes present in all the vertebrates but absent in the insects [1]. The number of vertebrate-specific genes is five times larger than that of insect-specific genes, thus indicating that vertebrates have more complex gene pools than insects. Some of these genes have been acquired in order to support insect- and vertebrate-specific characteristics during evolution. However, if some of these vertebrate genes are shared with lophotrochozoans (the third large superphylum of Bilateria), this would imply that they were present in the last common ancestor of Bilateria (Urbilateria) and have been lost from insects during evolution [2,3]. A number of genes categorized to this group have already been reported and characterized [4]. Meanwhile, if some of these insect genes are shared with lophotrochozoans, this would suggest that they were specifically acquired by the last common ancestor of protostomes but not deuterostomes. Such genes have not been reported to date.

The origin and evolution of the metazoan central nervous system (CNS) have been intensively discussed. Large-scale expression analysis of neural genes in hemichordates has revealed that the mediolateral patterning genes (Pax6, $d b x$, and $m s x$ ) and neural differentiation markers are expressed around the circumference of the embryo [5,6]. These results suggest that the centralization of a nervous system was acquired independently in deuterostomes and protostomes [7]. Meanwhile, several studies on the development of the CNS in Drosophila melanogaster and mouse have revealed common genetic patterning mechanisms in the formation of the insect and vertebrate brain. In both insects and vertebrates, the correct regionalization and neuronal identity of the anterior brain region is regulated by the cephalic gap genes otd/Otx and ems/Emx, whereas patterning of the posterior brain involves members of the Hox genes [8]. A recent study on gene expression patterns in the brain of developing annelids (Platynereis) has demonstrated that the patterning mechanism of the CNS is well conserved among chordates and annelids [9]. These studies strongly indicate that Urbilateria already had an anatomically complex CNS. Furthermore, cross-species comparisons of genome sequences and expressed sequence tag data sets have demonstrated the presence of a common ancestral CNS at the molecular level $[3,10]$. These results suggest that Urbilateria and the last common ancestor of protostomes were genetically complex, and have complex nervous systems [4].

Mushroom bodies (MBs) are lobed neuropils that comprise long and approximately parallel axons originating from clusters of minute basophilic cells located dorsally in the most anterior neuromere of the CNS. Structures with these morphological properties are found in many marine annelids (lophotrochozoa) and almost all arthropods (ecdysozoa) except crustaceans [11]. MBs are higher multisensory centers (for example, olfaction and vision) of the insect brain and are implicated in olfactory and other forms of associative learning [12]. There are two possibilities regarding the presence of $\mathrm{MBs}$ in different animal lineages (arthropod groups except crustacea and lophotrochozoa). One possibility is that the ancient MBlike structure was present in the CNS of the last common ancestor of all protostomes, and then some species have evolved the present MBs but the others have lost it secondarily during evolution. Another possibility is that MBs have independently evolved several times in different animal lineages by convergent evolution. It was reported that several genes encoding transcription factors, eyeless (ey), twin of eyeless (toy), and dachsund (dac), are necessary for the development of the Drosophila MB [13-15]. Their homologs are present in various metazoan genomes, and Pax6 (the vertebrate homolog of $e y$ ), for example, also has essential roles for neural development [16]. To prove the single origin of MBs, it will be necessary to demonstrate that the expression domains of the above genes are conserved in the arthropod and lophotrochozoan CNS during development.

Here, we report a novel gene, namely PsGEF (GEF, guanine nucleotide exchange factor), which is present in insect and Lottia (lophotrochozoa) genomes but absent in Nematostella (cnidaria), deuterostome, and nematode genomes. It is likely that PsGEF was specifically acquired by the last common ancestor of protostomes, and then lost in some species, for example nematodes. Intriguingly, the presence of PsGEF containing the C2, PDZ, and RhoGEF domains appears to coincide with the presence of MBs. Further, in Drosophila, PsGEF functions as a GEF for Rac and is essential for axon development in MBs. These results suggest that gain, retention, and loss of PsGEF are associated with some structural and/or functional features common between insect and lophotrochozoan nervous systems, which are absent in all deuterostomes and cnidarians. Thus, PsGEF is one of candidate genes associated with the diversity of metazoan nervous systems.

\section{Results \\ Identification of PsGEF gene uniquely shared between insects and limpets}

A large-scale comparison of the genomes of five insects (D. melanogaster, Anopheles gambiae, Aedes aegypti, Apis mellifera, and Tribolium castaneum) and five vertebrates (Homo sapiens, Mus musculus, Monodelphis domestica, Gallus gallus, and Tetraodon nigroviridis) revealed that there were approximately 1,000 insect-specific orthologous genes [1]. We searched among these genes for those that are 
highly expressed in the Drosophila CNS by screening transgenic lines in which GAL4 was inserted in the promoter regions of candidate genes, in order to understand the genetic basis for the development and functions specific for the insect nervous system. From the screening, we found one gene, the PsGEF gene (CG14045).

Drosophila PsGEF (DmPsGEF) encodes a protein with the $\mathrm{C} 2, \mathrm{PDZ}$, and RhoGEF domains (Figure 1B). The C2 domain is a $\mathrm{Ca}^{2+}$-dependent membrane-targeting module found in many proteins involved in signal transduction or membrane trafficking [17]. It is thought to be involved in $\mathrm{Ca}^{2+}$-dependent phospholipid binding and in membranetargeting processes [18]. The PDZ domain mediates binding with other proteins and is found in many signaling proteins frequently associated with the plasma membrane [19]. Moreover, it is often associated with scaffolding proteins important for synaptic development [20]. The RhoGEF domain activates Rho family GTPases, namely, Rho, Rac, and Cdc42, through release of bound guanosine diphosphate and subsequent binding of guanosine triphosphate [21]. Thus, RhoGEF activity of PsGEF appears to require an increase in the intracellular $\mathrm{Ca}^{2+}$ level as well as association of PsGEF with other proteins. As shown in Figures $1 \mathrm{~A}$ and $1 \mathrm{~B}$, two different DmPsGEF transcripts (short and long mRNAs consisting of four and seven exons, respectively) are found. The short mRNA encodes a 786-amino acid protein containing the $\mathrm{C} 2$ and PDZ domains, and the long mRNA encodes a 1493-amino acid protein containing the $\mathrm{C} 2, \mathrm{PDZ}$, and RhoGEF domains. These two types of DmPsGEF mRNAs appear to be synthesized by alternative polyadenylation; polyadenylation at the 3 ' end of exons 4 and 7 results in the synthesis of short and long DmPsGEF mRNAs, respectively (Figure 1A). The short PsGEF with C2 and PDZ domains exhibits significant similarity to the vertebrate RGS3, which also contains $\mathrm{C} 2$ and PDZ domains [22]. However, PsGEF lacks amino acid sequences necessary for constituting the regulator of G-protein signaling (RGS) domain together with the C2 and PDZ domains, thus suggesting that the short PsGEF does not function as an RGS.

$D M P s G E F$ orthologs are also present in the genomes of Nasonia vitripenis (parasitic wasp), Pediculus humanus corporis (human body louse), and Acyrthosiphon pisum (pea aphid). The proteins encoded by these contain $\mathrm{C} 2$, PDZ, and RhoGEF domains, similar to the long version of the DmPsGEF. PsGEF was therefore present in the common ancestor of holometabolous and hemimetabolous insects. Further, we searched for insect PsGEF orthologs in the genomes of Nematostella vectensis (sea anemone, cnidaria), Strongylocentrotus purpuratus (sea urchin), Ciona intestinalis (sea squirt), Lottia gigantea (limpet, lophotrochozoa), and Caenorhabditis elegans. We have found that only Lottia contains an insect PsGEF ortholog coding for a protein containing the $\mathrm{C} 2, \mathrm{PDZ}$, and RhoGEF domains. As PsGEF has a complex domain organization, it may be difficult to identify the orthologs if they contain very large introns in some cases. PsGEF is present in insects (ecdysozoa) and limpet (lophotrochozoa) but not sea anemone or deuterostomes, suggesting that PsGEF was specifically acquired by the last common ancestor of protostomes. Drosophila (DmPsGEF), Tribolium (TcPsGEF), Pediculus (PhcPsGEF), Apis (AmPsGEF), and Lottia (LgPsGEF) PsGEFs (Additional file 1) share the C2, PDZ, and RhoGEF domains as shown in Figure 1C. The alignment of amino acid sequences containing the above functional domains of five PsGEF proteins demonstrates that they show significant similarity only in the $\mathrm{C} 2, \mathrm{PDZ}$, and RhoGEF domains (Additional file 2). This was also the case when the full-length amino acid sequences were analyzed (data not shown).

The exon-intron organizations of the above five PsGEF genes are shown in Figure 2A. Insect and limpet PsGEFs contain one and two introns in the $\mathrm{C} 2$ domain-coding regions, respectively. Intriguingly, the position of one out of two introns is conserved at the same phase in five species (Figure 2B). Nevertheless, the size of this particular intron is varied, ranging from 562 to 2,482 base pairs. DmPsGEF has no intron, TcPsGEF and AmPsGEF have one intron, and PhcPsGEF and $L g P s G E F$ contain two introns in the PDZ domain-coding regions (Figure 2A). TCPsGEF, PhcPsGEF, and LgPsGEF share one intron position as well as phases among them (Figure 2C). DmPsGEF and LgPsGEF contain one intron, AmPsGEF has two introns, and TcPsGEF and PhcPsGEF contain four introns in the RhoGEF domain-coding regions (Figure 2A). Among them, one intron position is conserved at the same phase in $D m P s G E F$, TcPsGEF, and PhcPsGEF. In addition, the positions and phases of two introns are conserved in TCPsGEF and PhcPsGEF. Thus, TcPsGEF and PhcPsGEF share the same positions and phases of three out of four introns (Figure 2D). These results demonstrate that some but not all intron positions are conserved at the same phase in the $\mathrm{C} 2, \mathrm{PDZ}$, and RhoGEF domain-coding regions of five PsGEFs from different species.

\section{Daphnia PsGEF lacks RhoGEF domain}

We next analyzed the PsGEF ortholog in Daphnia pulex, which belongs to crustacea, one of major arthropod groups. The PsGEF ortholog is present in scaffold 53 of the Daphnia pulex genome assembly; however, the exons encoding the RhoGEF domain are apparently missing (Figure 3A). According to the JGI database [23], three predicted genes (SNAP_00018439, SNAP_00018441, and SNAP_00018442) are present in this genomic region. A SNAP_00018439 mRNA was detected by using reverse transcriptase-polymerase chain reaction (RT-PCR) and appears to encode the PsGEF protein containing only the 


\section{A}

$D m P s G E F$ short mRNA

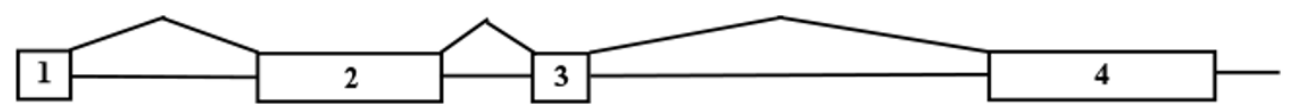

\section{$D M P s G E F$ long mRNA}

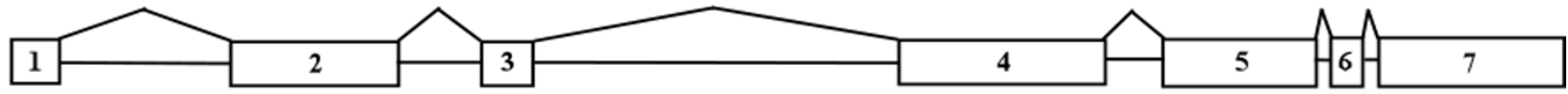

B

DmPsGEF short protein (786 amino acids)

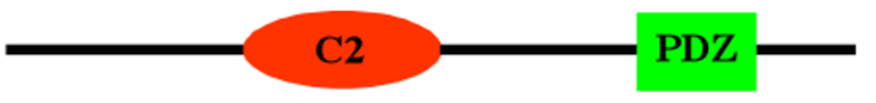

DmPsGEF long protein (1493 amino acids)

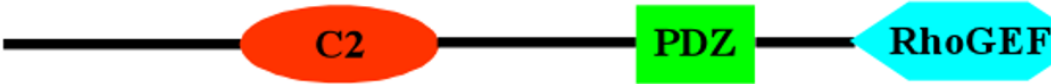

C

DmPsGEF 272

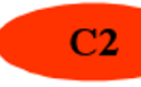

PDZ

RhoGEF 1132

TcPsGEF

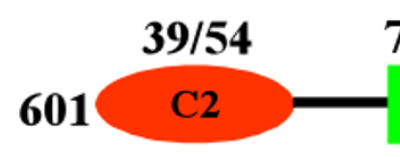

72/86 54/75

PDZ - RhoGEF 1313

PhcPsGeF

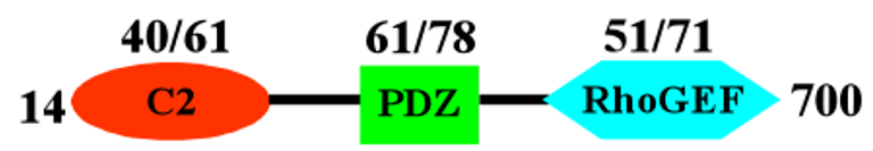

AmPsGEF

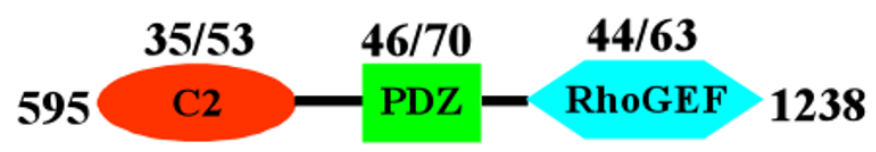

LgPsGEF

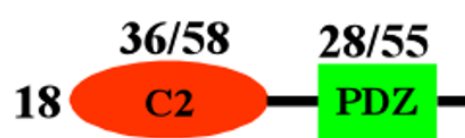

26/44

RhoGEF 730

Figure I (see legend on next page) 
Figure I (see previous page)

Structure of two different Drosophila PsGEF (DmPsGEF) mRNAs and proteins and comparison of C2, PDZ, and RhoGEF domains of 5 PsGEFs from different species. (A) Exon-intron organization and splicing pattern of DmPsGEF. The rectangles with numbers represent the exons, and the straight lines between the exons represent the introns. Polyadenylation at the $3^{\prime}$ ends of exon 4 and exon 7 results in the synthesis of short and long DmPsGEF mRNAs, respectively. (B) Domain organizations of DmPsGEF proteins. DmPsGEF short protein with 786 amino acids contains the C2 (red oval) and PDZ (green rectangle) domains, and DmPsGEF long protein with 1493 amino acids also contains the RhoGEF domain in addition (blue hexagon). (C) Comparison of C2, PDZ, and RhoGEF domains of DmPsGEF, TcPsGEF (Tribolium PsGEF), PhcPsGEF (Pediculus PsGEF), AmPsGEF (Apis PsGEF), and LgPsGEF (Lottia PsGEF). The amino acid sequences containing the C2, PDZ, and RhoGEF domains of TcPsGEF (amino acid 60I to I3I3), PhcPsGEF (amino acid I4 to 700), AmPsGEF (amino acid 595-I238), and LgPsGEF (amino acid 18 to 730) proteins are compared with those of DmPsGEF (amino acid 272 to II32). The numbers above C2, PDZ, and RhoGEF domains of TcPsGEF, PhcPsGEF, AmPsGEF, and LgPsGEF proteins represent \% identity/\% similarity of their amino acid sequences to those of DmPsGEF. See also Additional file I for the full-length amino acid sequences of five PsGEF proteins and Additional file 2 for alignment of above amino acid sequences.

C2 and PDZ domains. SNAP_00018442, but not SNAP_00018441 mRNA was detected by RT-PCR, and it encodes a novel protein with a PH-like domain (Figure 3B).

The above results indicate that PsGEF with the C2, PDZ, and RhoGEF domains is specifically present in lophotrochozoan and insect genomes but not in crustacean genomes. This distribution among metazoans accurately coincides with the presence of MBs, prominent neuropils involved in processing multiple sensory inputs as well as associative learning in the insect, platyhelminth, and annelid brains [11]. We therefore tested whether PsGEF plays a role in the development or functions of MB in Drosophila.

\section{Region-specific expression of DmPsGEF in the embryonic and adult central nervous system}

We identified several GAL4 enhancer trap lines (NP5114, NP0264, NP1088, NP7169, NP3316, NP7265, NP3612, and NP3237) located in the promoter region of DmPsGEF. These GAL4 lines were crossed with UAS-mCD8-GFP lines to detect the expression of DmPsGEF in embryos and adults, and all of them exhibited the same expression patterns. DmPsGEF is expressed in the subsets of cells in the brain and ventral nerve cord as well as cells at the midgut fusion point in the stage-15 embryos (Figures 4A and 4B). $D m P s G E F$ is highly expressed in MBs, the antennal lobe, and the optic lobe of the adult brain. In addition, there are several large discrete DmPsGEF-positive cells surrounding the antennal lobe (Figure 4C). The presence of DMPsGEFpositive lobes of MBs, as observed by staining for Fasciclin II (Fas II), suggested that DmPsGEF is expressed in the alpha/beta neurons of MBs (Figure 4D). AmPsGEF mRNA is also highly expressed in the adult honey bee brain (data not shown).

\section{DmPsGEF is necessary for the axon development of mushroom bodies}

To understand the functions of DmPsGEF, we generated DmPsGEF loss-of-function mutants by imprecise excision of NP5114. Two deletion mutants were recovered. The deletion mutants included $d m P s G E F \Delta 55$, in which $1.5 \mathrm{~kb}$ genomic DNA containing the promoter region is deleted, and $d m P s G E F{ }^{\Delta 21}$, in which 2.7 kb genomic DNA containing exons 1 and 2 is deleted (Figure 5A). Both $d m P s G E F \Delta 55$ and $d m P s G E F^{\triangle 21}$ are viable and fertile with no morphological defects. The expression of DmPsGEF mRNA was examined in NP5114, dmPsGEF ${ }^{\Delta 55}$, and $d m P s G E F^{\Delta 21}$ embryos by RT-PCR. Both short and long mRNAs are expressed in the NP5114 embryos; however, they are absent in $d m P s G E F \triangle 55$ and $d m P s G E F^{\Delta 21}$ embryos. The adjacent CG14047 mRNA is equally expressed in all embryos (Figure $5 \mathrm{~B}$ ). These results suggest that both $d m P s G E F \Delta 55$ and $d m P s G E F^{\Delta 21}$ are DmPsGEF-null alleles.

Since DmPsGEF is highly expressed in the alpha/beta neurons of MBs (Figures 4C and 4D), we analyzed the morphology of the alpha/beta lobes in $d m P s G E F \Delta 21$ hemizygous males by immunostaining of Fas II. The Fas II-positive alpha/beta lobes are thinner in $d m P s G E F \Delta 21$ than in wild-type males (Figures 6A and 6B). Furthermore, the alpha lobes of late-born alpha/beta neurons visualized by $201 Y$-GAL4 and UAS-mCD8-GFP are thinner in $d m P s G E F^{\Delta 21}$ because the alpha lobes are often short, and their positions along the beta lobes vary (Figures 6C to $6 \mathrm{E}$ ). The same results were obtained for $d m \mathrm{PsGEF}^{\Delta 55}$ (data not shown). We also analyzed the morphology of the alpha'/beta' lobes in $d m P s G E F^{\Delta 21}$ and wild-type males by anti-Trio antibody staining [24]. No significant difference was observed (data not shown).

For a more accurate analysis of the role of DmPsGEF in the development of MBs, the morphology of alpha/beta neurons at a single-cell level was examined by using the mosaic analysis with a repressible cell marker (MARCM) 
A
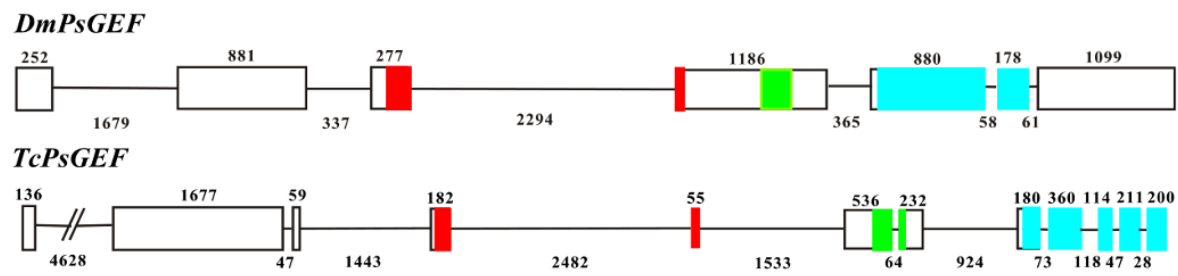

PhcPsGEF

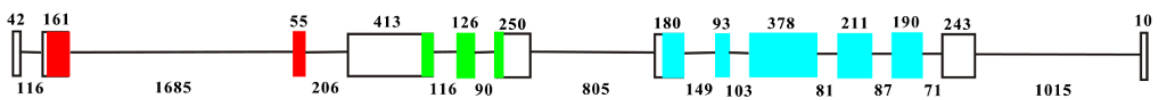

AmPSGEF

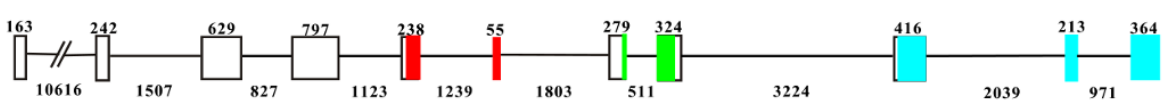

LgPsGEF

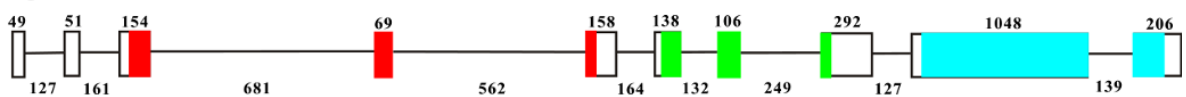

B

DmPsGEF RTAVHRHSGRPYFDQRFNF*QISSG---EETAGQYLQLAVWHRDRHLK2RSEFLGCSTFPLNELVHPD

TCPsGEF RTAVQADSSKPFFNHTFKF*PLQ----KESLQKRLHIEVWHRDRSSR2TSEFLGCMSFDVRHVYTKD

PhCPSGEF RTPVHRESSCPNFNETFSF*NFHS-_-_-EDL NKHULVSVWHRDRDNR2KSEFLGCVSLAVKNATKKE

AmPSGEF RTAVHRATPNPVFHETLIL*PCPVNNNHCSSKPTSLDIAVWHRDRRAR2RSELLGCWTLPLPLSQDKR

C

DmPsGEF LTRTEE-*RGFGFSIVWTHPPR*VEKIEAG*LSADRGG ILPGDYVIFVDKHNVVTMPEADVLNLIR*SQGSSLTLETFR

TCPSGEF LTRAPK- *SGFGFSVVWTHPPR*IERVEKG*LPAEKAGILPGDYI IFVDKHNVVMUPEIDILNLIR2SYGSQLTLEIFR

PhCPsGEF LTKQPG-*GGFGFSTANVQPPR2VERVEAG*QPADRAGTRPGDFVIFVDRFNVVTNSEEEILDI TK2SCGNELTLETYR

AmPsGEF LIRQTANQOTPMGFSLSWGRPPR*VERVDPG*SPAERSGLRPGDHVVFVDNTNVVTRSREEILGLTQ*AATNQL ILEVYR

LgPSGEF RGKN- *-GFGFSVVESFPVIK*VGRVDGAISPAEEAGLIRGDTTVKVNGQVVSRSTAVSVAKLLVK2RSSNKLVLDVQR

D

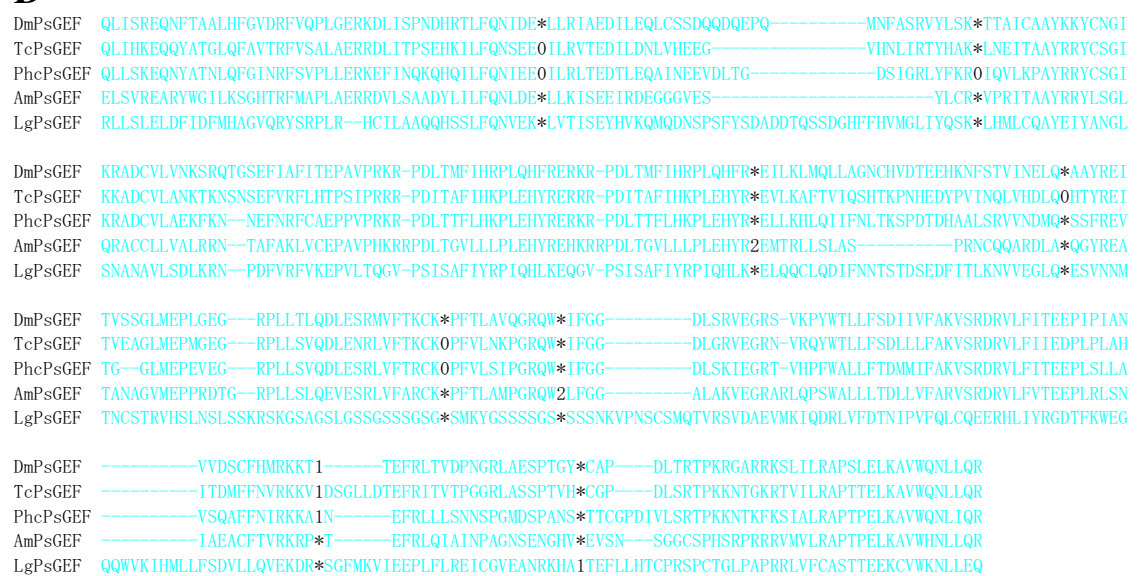

Figure 2

Exon-intron organizations as well as intron positions of five PsGEFs from different species. (A) Exon-intron organizations of DmPsGEF, TcPsGEF, PhcPsGEF, AmPsGEF, and $L g P s G E F$. The exon and intron are represented by rectangle and straight line, respectively. Exon I is on the left. The numbers above exons and below introns indicate their sizes, and thus their scales are different among five PsGEFs. The exon sequences encoding the C2, PDZ, and RhoGEF domains are highlighted by red, green, and blue, respectively. The initiation codon is present in the exon I except DmPsGEF in which it is located in the exon 2. (B to D) Intron positions within the C2 (B), PDZ (C), and RhoGEF (D) domain-coding regions of DmPsGEF, TcPsGEF, PhcPsGEF, $A m P s G E F$, and $L g P s G E F$ are indicated by digits corresponding to the phase of the intron relative to the surrounding codons (phase $0, \mathrm{I}$, and 2 introns fall before the first, second, and third bases of a codon, respectively). Asterisk indicates the absence of intron. Some intron positions are conserved at the exact homologous positions and phases between five PsGEFs. 

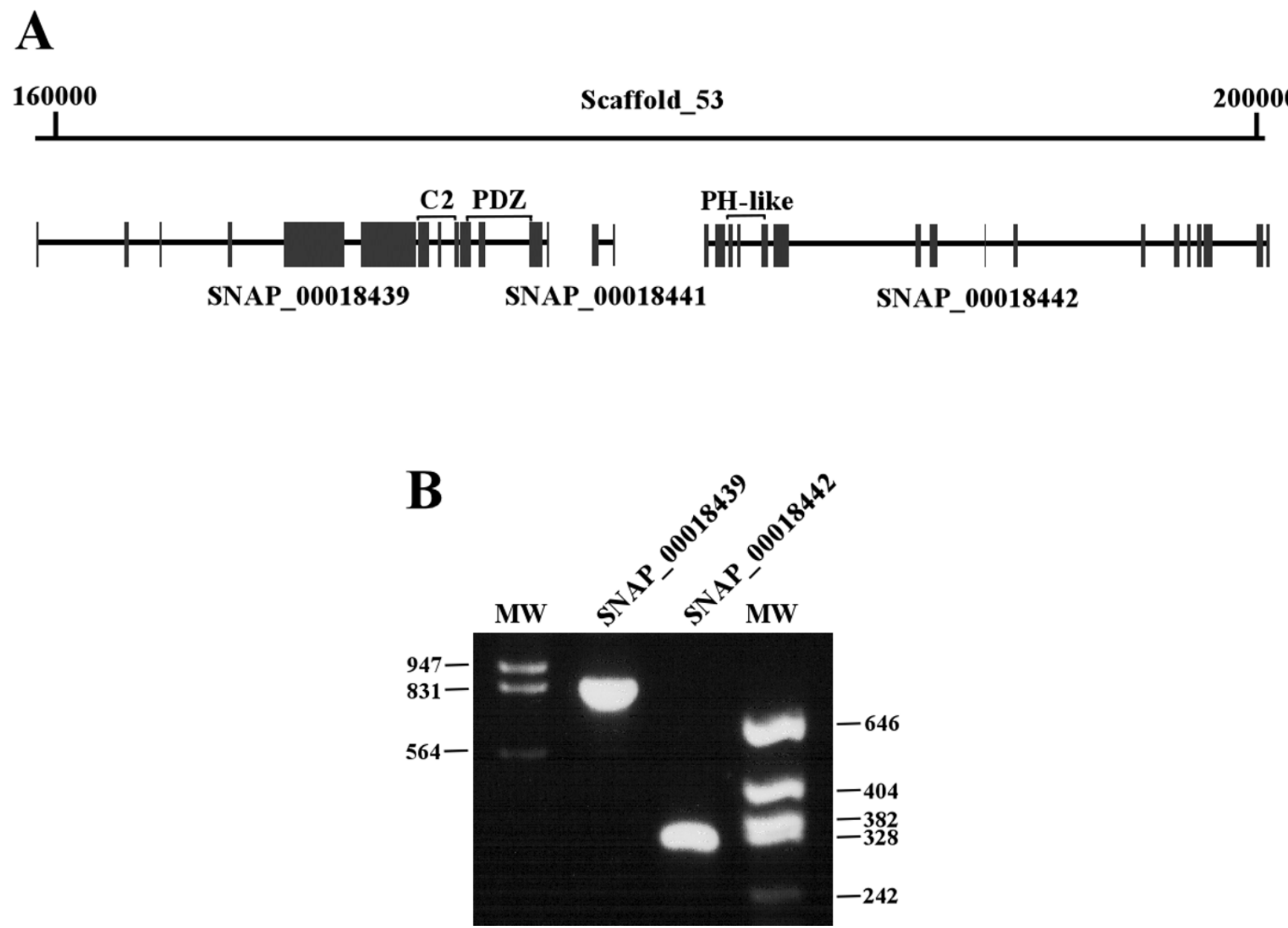

Figure 3

Daphnia pulex genomic DNA region containing PsGEF and mRNA expression of Daphnia PsGEF and neighboring genes. (A) Daphnia PsGEF is located in scaffold 53 of the genome assembly. The predicted transcript SNAP_00018439 encodes PsGEF containing the C2 and PDZ domains. The neighboring transcripts SNAP_0001844I and SNAP_00018442 encode a novel short protein and a protein with a $\mathrm{PH}$-like domain, respectively. The predicted exons are indicated by the solid rectangles. The exons encoding the $\mathrm{C} 2, \mathrm{PDZ}$, and $\mathrm{PH}$-like domains are indicated by brackets. The exons encoding the RhoGEF domain are not present in this scaffold. (B) The expression of SNAP_000I8439and SNAP_000/8442 mRNAs in Daphnia is confirmed by using RT-PCR. However, SNAP_000/844I mRNA can not be detected by this analysis. The numbers at both sides of the panel indicate the sizes of bands in molecular weight markers (MW).

system [25]. The wild-type alpha/beta neurons bifurcate their axons into the alpha and beta lobes (Figure 6F); however, $34 \%$ of late-born $d m P s G E F^{\Delta 21}$ alpha/beta neurons $(n=35)$ have branching defects, and the axons toward the alpha lobe are missing (Figures 6G and 6H). These results demonstrate that DmPsGEF is necessary for the correct axonal development of alpha/beta neurons in MBs.

\section{DmPsGEF genetically interacts with Racl but not other Rho family members for the axon development in mushroom bodies}

As PsGEF is expected to function as a RhoGEF, we analyzed the genetic interactions of DmPsGEF with Rho family members. We thus examined the morphology of the alpha/beta lobes in the MBs of $d m P s G E F^{\Delta 21}$ hemizygous males in a $r h o 1^{E 3.10}, \operatorname{rac}^{111}, \operatorname{rac}^{\Delta}{ }^{\Delta}$, and $m t^{\Delta}$ heterozygous background. For the interaction with $C d c 42, d m P s G E F \Delta 21$ homozygous females with a $c d c 42^{4}$ heterozygous background were analyzed. It was observed that reducing the gene dosages of $C d c 42$, Rho1, Rac2, and Mtl does not affect the phenotypes of the alpha/beta lobes as observed with 

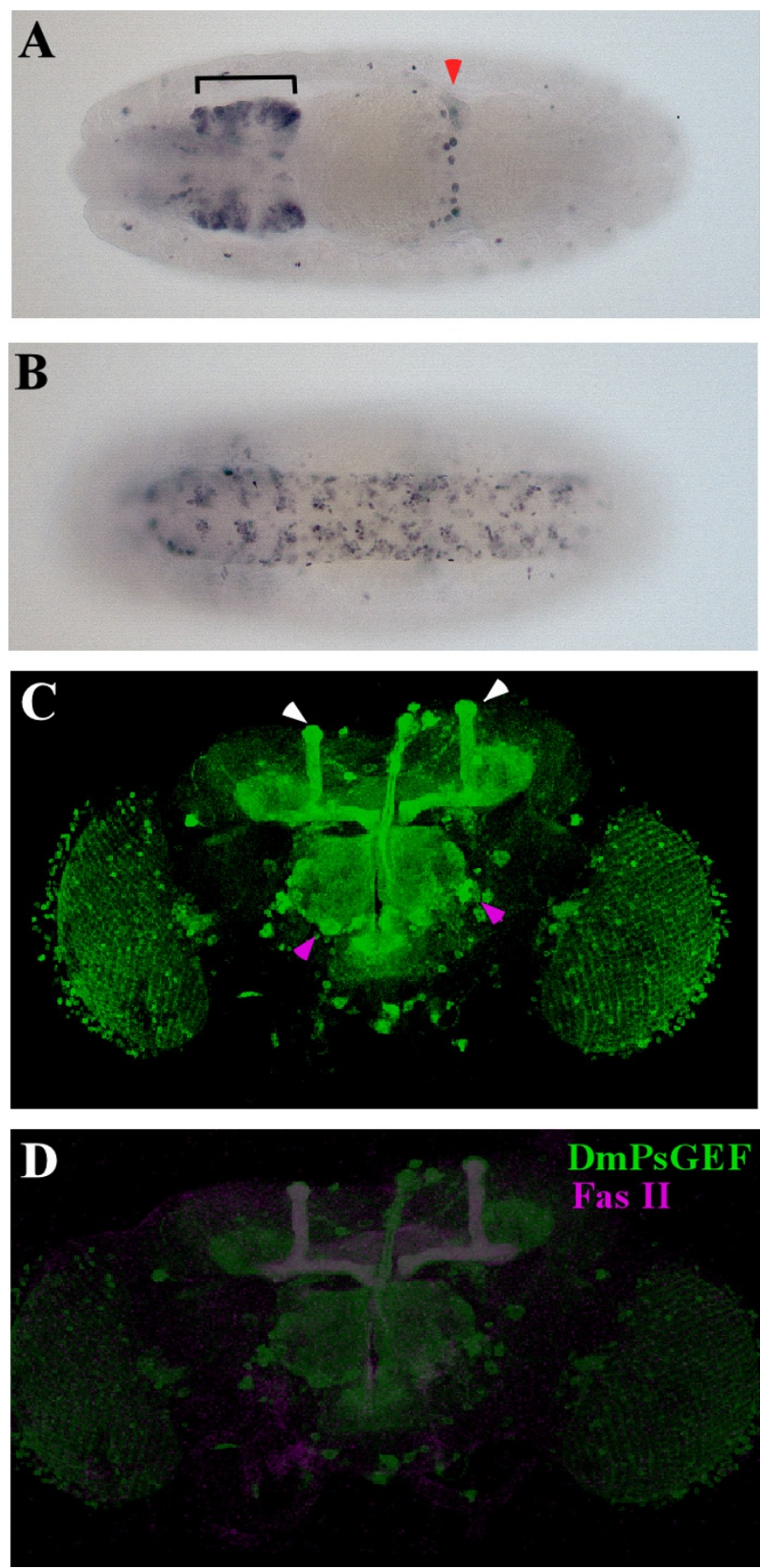

Figure 4

Expression patterns of DmPsGEF in Drosophila. (A to D) The expression of DmPsGEF is examined in progenies carrying NP5 I I 4 and UAS-mCD8::GFP. DmPsGEF is expressed in the subsets of cells in the brain (A, bracket) and the ventral nerve cord (B) as well as cells at the midgut fusion point (A, red arrowhead) of the stage- 15 embryos. The dorsal and ventral views are shown in (A) and (B), respectively. The anterior view is on the left. DmPsGEF is expressed in the optic lobes, antennal lobes, discrete neurons surrounding the antennal lobes ( $C$, magenta arrowheads), and mushroom bodies (C, white arrowheads) in the adult brain. DmPsGEF (indicated in green) is primarily expressed in Fas Il-positive alpha/beta neurons (shown by magenta) in mushroom bodies (MBs) (D). 


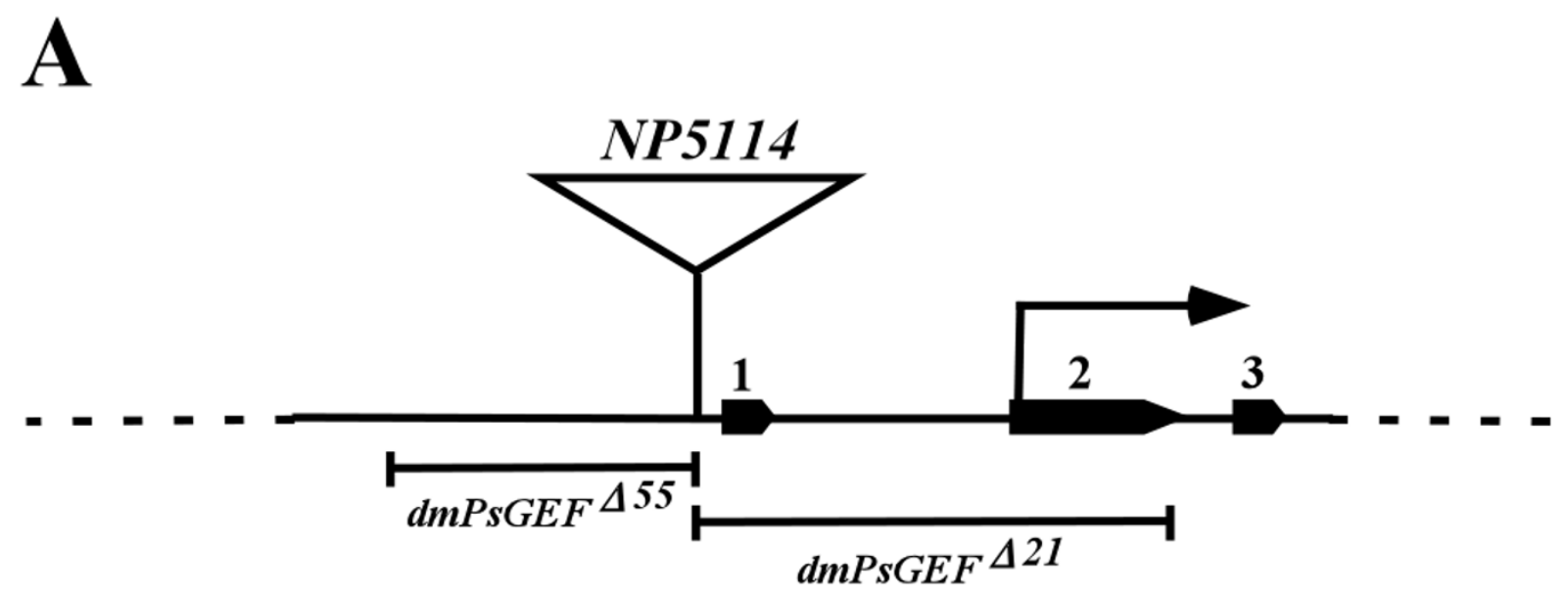

1 kb

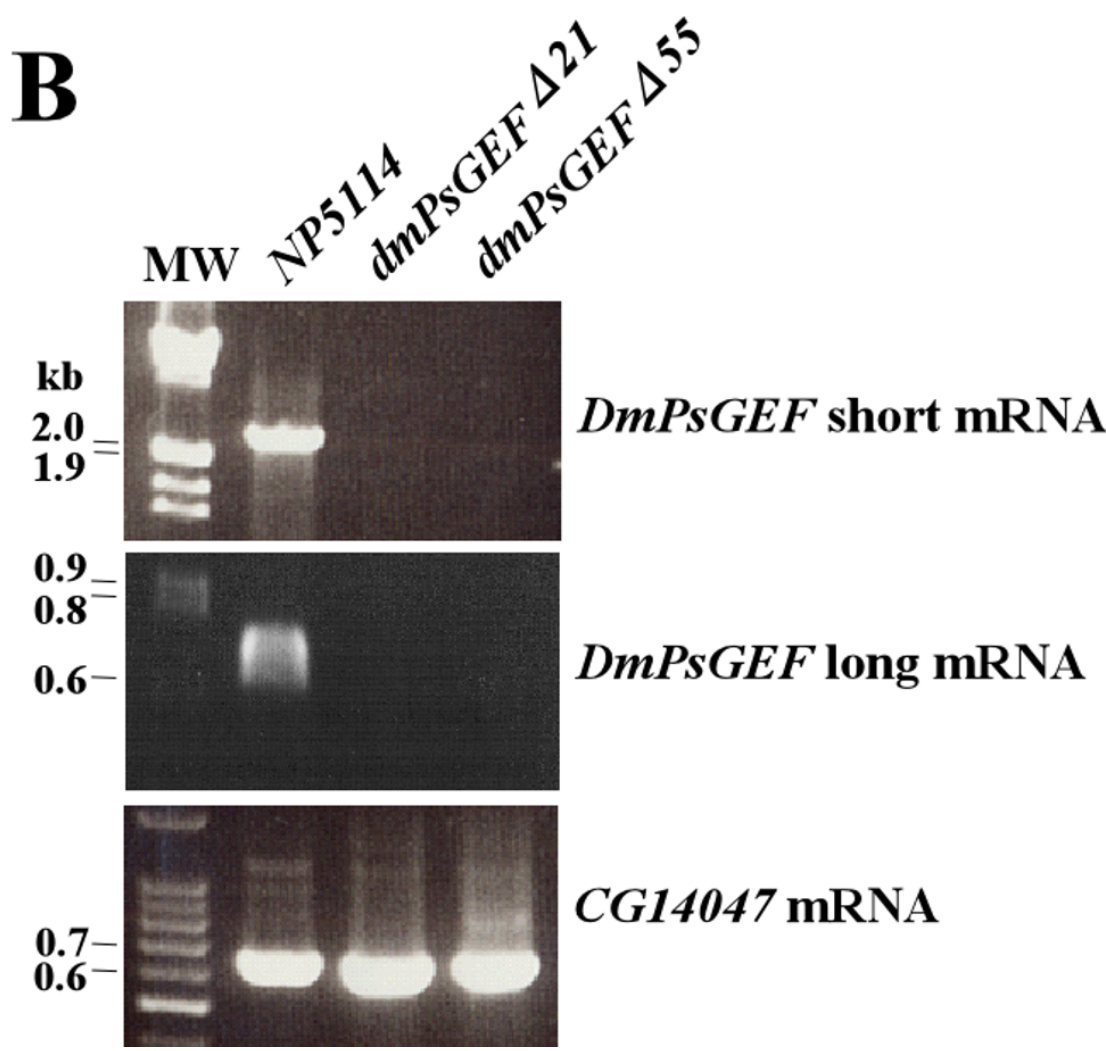

Figure 5

Generation of DmPsGEF deletion mutants and DmPsGEF mRNA expression analysis in the mutants. (A) NP5 I / 4 is located at $5^{\prime}$ upstream of exon I of DmPsGEF, and translation is initiated at exon 2 (arrow). Exons I to 3 are indicated by solid pentagons. Two DmPsGEF deletion mutants ( $\mathrm{dmPs}_{\mathrm{S}} \mathrm{GEF} \triangle 55$ and $\mathrm{dmPs} G E F \Delta 2 l$ ) were generated by imprecise excision of NP5 I /4, and the deleted genomic region in each mutant is also shown. The scale bar indicates I kb. (B) The expression of short and long DmPsGEF mRNAs as well as CG 4047 mRNA in NP5 I /4, dmPsGEF ${ }^{\Delta 2 I}$, and dmPsGEF ${ }^{\Delta 5}$ embryos was examined by reverse transcriptase-polymerase chain reaction. Both short and long DmPsGEF mRNAs are present in the parent NP5 I I 4 but not in $d m P s G E F \triangle 2 I$ and $d m P s G E F \triangle 55$ embryos, while CGI 4047 mRNA is present in all genotypes of embryos. The numbers at left side of the panels indicate the sizes of bands (in $\mathrm{kb}$ ) in molecular weight markers (MW). 

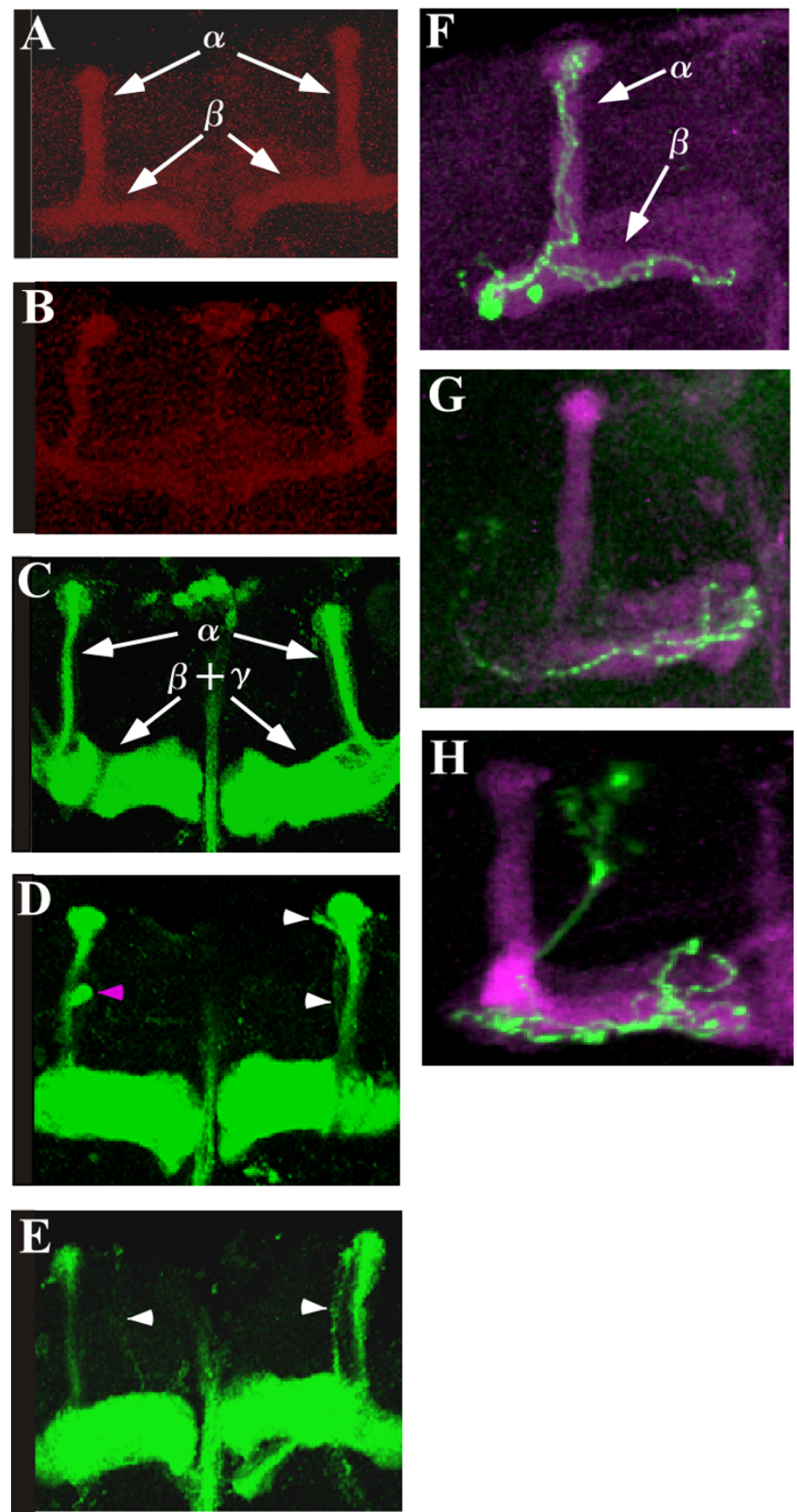

\section{Figure 6}

Morphology of alpha/beta lobes and alpha/beta neurons in mushroom bodies of the wild-type and the

dmPsGEF $\triangle 21$ mutant. The alpha lobes detected by Fas II staining are thinner in the dmPsGEF 221 mutants (B) than in the wildtype (A). The alpha and beta lobes are indicated by arrows in (A). The alpha lobes of late-born alpha/beta neurons are visualized by 20/Y-Gal4 and UAS-mCD8::GFP in the wild-type (C) and dmPsGEFA2I mutants (D and E). The alpha lobes are thinner in dmPsGEF 221 mutant than in the wild-type, as observed above. In addition, the alpha lobes are often short (magenta arrowhead in $\mathrm{D}$ ), and their positions along the beta lobes vary (white arrowheads in $\mathrm{D}$ and $\mathrm{E}$ ). The alpha as well as the beta and gamma lobes are indicated by arrows in (C). Findings of the repressible cell marker analysis reveal that the axons of wild-type alpha/ beta neurons (green) bifurcated toward the alpha/beta lobes (magenta) (F). However, the axons of $\mathrm{dmPs} G E F \Delta 2 I$ alpha/beta neurons (green) often fail to grow toward the alpha lobes ( $\mathbf{G}$ and $\mathbf{H})$. The alpha and beta lobes are indicated by arrows in (F). 
dmPsGEF ${ }^{\Delta 21}$ (Figures 7A and 7C to 7G). However, reduction in the Rac1 gene dosage dramatically influences the phenotypes: a pair of alpha/beta lobes was missing in $90 \%$ of the animals examined (Figure $7 \mathrm{H}$ ). It was observed that $65 \%$ of $r a c 1^{J 11} /+$ heterozygotes have normal alpha/beta lobes (Figure 7B), and 35\% have the branching defects as previously reported [26]. These results demonstrate that DmPsGEF genetically interacts with Rac1. However, DmPsGEF does not exhibit genetic interaction with Pak, which is a downstream effector of Rac1 (Figure 7I). This suggests that DmPsGEF activates Rac1; the active GTP. Rac1 is involved in the axon development in MB neurons via a Pak-independent signaling pathway.

\section{DmPsGEF functions as a GEF for Rac in cultured cells} To test whether PsGEF is a GEF for Rac, the RhoGEF domain of DmPsGEF was expressed in HeLa cells, and the F-actin of these cells was visualized by using fluorescein isothiocyanate (FITC)-phalloidin. The RhoGEF activity of PsGEF is likely to be affected by the intracellular $\mathrm{Ca}^{2+}$ level as well as its interaction with other proteins through the C2 and PDZ domains. Thus, only the RhoGEF domain of DmPsGEF was expressed in the HeLa cells. Actin polymerization in membranes resulting in membrane ruffling is specifically observed in the cells expressing the RhoGEF domain of DmPsGEF (Figures 8C and 8F). These phenotypes are similar to those obtained with the expression of active forms of Rac but not Cdc42 or Rho [27]. The active forms of Cdc42 and Rho are known to induce filopodia and stress fibers, respectively [28]. These results therefore suggest that the RhoGEF domain of DmPsGEF activates Rac but not Cdc42 or Rho in cultured cells. This finding is consistent with the genetic interaction of DmPsGEF with Rac1 but not $C d c 42$ or Rho1, as described earlier.

\section{Discussion \\ Genes specifically necessary for the development andlor functions of the insect nervous system}

We are interested in discovering the specific molecular features underlying the functions of insect nervous systems. There are several properties specific to the insect nervous system. One of the characteristics of the holometabolous insect brain is the development of the brain from the larval to the adult stages during metamorphosis. Although the overall brain organization is preserved during metamorphosis, dramatic remodeling of neuronal circuits occurs. Some embryonic and early larval neurons die, and others undergo axon pruning and regrowth. Furthermore, many neurons are born during metamorphosis [29]. The genes involved in neuronal remodeling and metamorphosis might be specific to the insect genomes. With regard to the structural characteristics of the insect brain, MBs are specific neuropil structures found in many marine annelids and almost all arthropod groups, except crustaceans [11]. MBs are lobed neuropils that comprise long and approximately parallel axons originating from clusters of minute basophilic cells located dorsally in the most anterior neuromere of the CNS. MBs are higher olfactory and multisensory centers of the insect brain and are implicated in odor discrimination and in olfactory and other forms of associative learning [12]. It is not known how MBs evolved in different taxa. There may be some common genes among annelids and insects, and they may have a role in the evolution of MBs. PsGEF may be one of these genes.

\section{Origin and evolution of PsGEF}

We first identified PsGEF as an insect-specific gene, which is highly expressed in the Drosophila CNS. It is present in the sequenced genomes of insects but not vertebrates. A search for PsGEF orthologs in other sequenced genomes revealed that $P S G E F$ is present in the parasitic wasp, the human body louse, and, surprisingly, the limpet Lottia. Due to the phylogenetic distance between insects and limpets, it is unlikely that PsGEF independently evolved in these taxa. In fact, a domain-by-domain phylogenetic analysis of RhoGEFs from different species demonstrates that the C2, PDZ, and RhoGEF domains of LgPsGEF cluster with those of insect PsGEFs, and do not branch with those of different families (data not shown). Thus, it is unlikely that PsGEFs have been independently generated by exon shuffling (or exon capture) in the lophotrochozoan and insect lineages. The exon-intron structures of five PsGEFs from different species also support this conclusion since the positions as well as phases of some introns are conserved in the $\mathrm{C} 2, \mathrm{PDZ}$, and RhoGEF domain-coding regions (Figure 2). This may suggest that the last common ancestor of protostomes gained the intron-rich ancestor of $P S G E F$, and some of these ancient introns have been lost, and some new introns have been gained in each species, as demonstrated with, for example, nuclear OXPHOS genes [30].

Apparently, intron loss has been most extensive in DmPsGEF. PsGEF was absent in Urbilateria, and then specifically acquired by the common ancestor of all protostomes but not deuterostomes or cnidarians. How was the ancient PsGEF originated? Since vertebrates possess RGS3 with $\mathrm{C} 2$ and PDZ domains similar to the short form of DmPsGEF, this gene was present in Urbilateria. It then acquired the exons encoding the RhoGEF domain by exon shuffling or exon capture from the different families to generate PsGEF [31]. Intriguingly, PsGEF is absent in nematodes, suggesting that it has been secondarily lost from some ecdysozoan species. Since it has been demonstrated that the evolutionary rates of insects and nematodes are fast and comparable [2,3,32], it is difficult to imagine that nematodes evolved an alternative pathway or paralogs of PsGEF to compensate for the absence of PsGEF and insects failed to evolve such systems. A more plausible explana- 

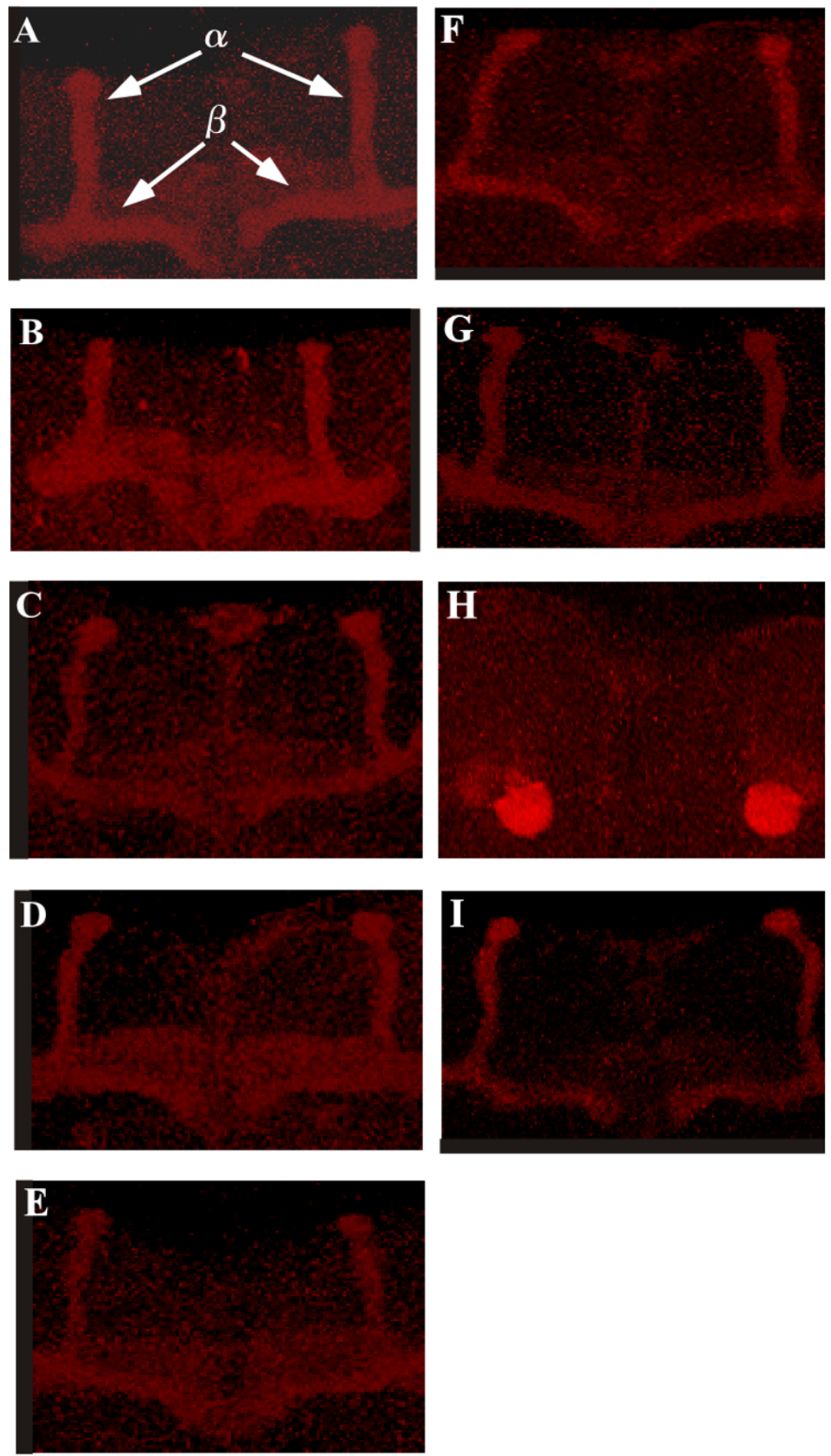

Figure 7

Genetic interaction of DmPsGEF with Rho family members. The morphology of the alpha/beta lobes of mushroom

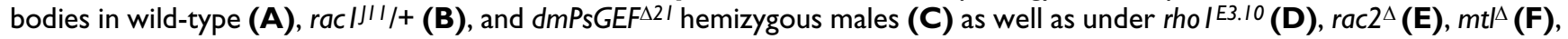
$\operatorname{racl}^{\prime \prime \prime}(\mathbf{H})$, and $\operatorname{pak}^{6}(\mathbf{I})$ heterozygous background is shown. To determine the interactions with Cdc42 (G), dmPsGEF $\Delta 21$ homozygous females with $c d c 42^{4}$ heterozygous background were analyzed. The alpha and beta lobes are indicated by arrows in (A). The reduction of Racl gene dosage dramatically influences the phenotypes; a pair of alpha/beta lobes is missing in $90 \%$ of the examined animals $(\mathrm{H})$. 

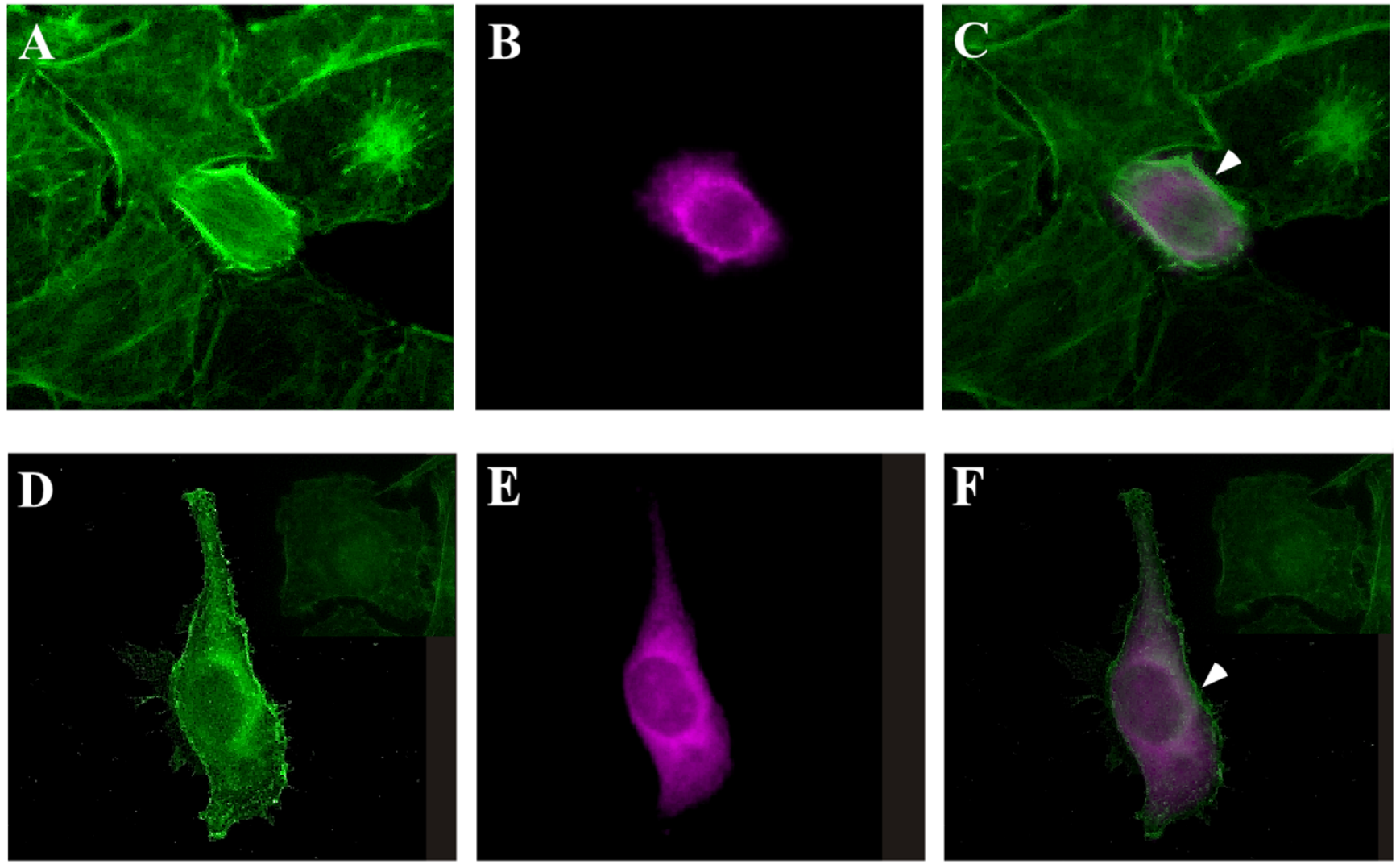

\section{Figure 8}

PsGEF functions as a GEF for Rac in cultured cells. HeLa cells transiently transfected with DmPsGEF RhoGEF domain expression constructs were stained with fluorescein isothiocyanate-phalloidin to detect F-actin (A and D), and anti-myc antibodies were used to detect the RhoGEF domain of DmPsGEF (B and E). The images $A$ and $B$ are merged to form image (C), and the images $D$ and $E$ are merged to form image (F). Actin polymerization in membranes, which results in membrane ruffling, is specifically observed in cells that express the RhoGEF domain of DmPsGEF (arrowheads in C and F).

tion is that PsGEF has been selectively retained in the insect and limpet genomes because it continues to play an important role in these animals but not in nematodes.

An analysis of the Daphnia genome revealed that the Daphnia PsGEF contains only the C2 and PDZ domains, and it corresponds to the short form of DmPsGEF. The exons encoding RhoGEF domains are apparently missing in the Daphnia genome (Figure 3). This demonstrates that the exons encoding the RhoGEF domain were specifically deleted in crustaceans after split from a common ancestor of crustaceans and insects [33,34]. These results suggest that Daphnia PsGEF cannot function as a RhoGEF, which is critical for $\mathrm{MB}$ axon development (Figure 7 and see below). Thus, PsGEF containing the C2, PDZ, and RhoGEF domains appears to be present only in the limpet Lottia and insects but not in crustaceans. Although it is not known whether MB-like structures are present in the limpet brain, this distribution pattern among metazoans coincides with the presence of MBs in the brains of certain platyhelminthes, marine annelids, and insects but not crustaceans [35]. These results suggest that PsGEF may be associated with the evolution of MB-like brain structures and is, in fact, necessary for $\mathrm{MB}$ axon development in Drosophila (Figure 6).

\section{Molecular functions of PsGEF}

Alternative splicing of DmPsGEF determines the presence or absence of the RhoGEF domain in the protein (Figures $1 \mathrm{~A}$ and $1 \mathrm{~B}$ ). The activation of Rac by PsGEF is therefore directly regulated by alternative splicing. What are the functions of the short version of PsGEF with only the C2 and PDZ domains, which is also present in Daphnia? If both short and long PsGEFs are present in a single neuron, 
the short protein may exert dominant negative effects on the long protein. The short and long proteins may compete for binding with $\mathrm{Ca}^{2+}$ and certain proteins through the $\mathrm{C} 2$ and PDZ domains, respectively. This, in turn, regulates the RhoGEF activity of the long PsGEF. If their expression is mutually exclusive, they may have independent functions in different neurons. Since it is difficult to distinguish the expression patterns of short and long DmPsGEF mRNAs in Drosophila, an examination of the functions of short PsGEF in Daphnia may provide an answer to the abovementioned question. Although alternative splicing of PsGEF occurs in the fruit fly and honey bee (data not shown), it remains to be established whether the same alternative splicing occurs in other insects and limpets.

Loss of DmPsGEF results in thinner alpha lobes than those of wild-type (Figures 6 and 7). Furthermore, short and multiple alpha lobes derived from late-born 201Y-Gal4expressing alpha/beta neurons were observed in MBs of $d m P s G E F^{\Delta 21}$ flies, as shown in Figures $6 \mathrm{C}$ to 6E. These results suggest that the alpha/beta neurons bifurcate their axons at more random positions in $d m P s G E F^{\Delta 21}$ flies. Further, the $d m P s G E F^{\Delta 21}$ alpha/beta neurons may not respond well to the signals provided by guidance neurons (the pre-existing alpha/beta neurons). Moreover, some alpha/beta neurons fail to extend their alpha-axonal branches (Figures $6 \mathrm{~F}$ to $6 \mathrm{H}$ ). Meanwhile, gamma neurons appear to be normal. Alpha'/beta' neurons are generally born during the late third instar, and their axons (alpha'/ beta' lobes) remain intact during metamorphosis [25]. These lobes may serve as the guiding axons for the bifurcation of the axons of alpha/beta neurons. We therefore

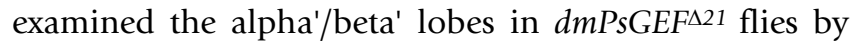
immunostaining with anti-Trio antibody [24]. Their morphology was found to be normal, thus suggesting that $D m P s G E F$ is specifically necessary for the axonal development of alpha/beta neurons in MBs.

To identify Rho family members activated by PsGEF, we first tested the genetic interaction of DMPsGEF with five Rho family members. The results indicated that DmPsGEF genetically interacts with Rac1 but not other family members (Figure 7). Consistent with this observation, the ectopic expression of the RhoGEF domain of DmPsGEF induces actin polymerization in the membrane of HeLa cells (Figure 8). These results thus suggest that PsGEF activates Rac but not Cdc42 or Rho in vivo. Intriguingly, DMPSGEF does not genetically interact with Pak, one of the downstream effectors of Rac (Figure 7I). Trio was shown to activate Rac and promote LIM kinase activity via Pak to induce axon growth inhibition [36]. Thus, DmPsGEF induces Rac activation and may stimulate axon growth via a Pak-independent pathway along with Still life (Sif) [36,37]. Three GEFs for Rac (Trio, Sif, and DmPs-
GEF) appear to function for the morphogenesis of MBs in Drosophila. Since each GEF protein has specific functional domains (for example, Spectrin repeats and SH3 domain in Trio, PH and PDZ domains in Sif) in addition to the RhoGEF domain, the mechanism to activate individual GEFs should be different. Specifically, the presence of the C2 domain in PsGEF suggests that the increase of intracellular $\mathrm{Ca}^{2+}$ level is essential for the localization of PsGEF at the plasma membrane where Rac proteins are anchored. The function of DmPsGEF is therefore dependent on the local increase of cytosolic $\mathrm{Ca}^{2+}$ level which occurs during the axonal development of alpha/beta neurons in MBs.

$D m P s G E F$ is expressed not only in the MBs but also in the antennal and optic lobes in adult Drosophila brain. We analyzed the axonal projection patterns of several olfactory neurons into the antennal lobes in $d m P s G E F^{\Delta 21}$ flies; however, no significant difference was observed relative to the patterns observed in the wild-type (data not shown). The glomerular structures of the antennal lobes visualized by immunostaining with anti-nc 82 antibody were found to be normal (data not shown). Although we did not observe gross morphological defects in the antennal and optic lobes in $d m P s G E F^{\Delta 21}$ flies, it is possible that there were subtle defects in these brain regions. No other proteins with $\mathrm{C} 2$ and PDZ domains are present besides DmPsGEF in Drosophila. However, other GEFs for Rac (Trio and Sif) could be partially redundant with DmPsGEF. This may explain the lack of phenotypes associated with the antennal lobes in $d m P s G E F^{\Delta 21}$ flies. We are also testing the olfactory and visual behaviors of adult $d m P s G E F^{\Delta 21}$ flies at present.

\section{Association of PsGEF with structural and/or functional features common between insect and lophotrochozoan nervous systems}

The specific acquisition of PsGEF by the last common ancestor of protostomes followed by the retention or loss in specific animal species during evolution demonstrates that there are some structural and/or functional features common between insect and lophotrochozoan nervous systems, which are absent in all deuterostomes and cnidarians. We would like to propose that PsGEF may be associated with the presence of MBs, specific brain structures in insects, annelids, and platyhelminthes [35]. The fact that PsGEF containing the C2, PDZ, and RhoGEF domains is specifically retained in animals having MBs (for example, insects but not crustaceans in arthropods) in addition to functioning as a GEF for Rac, which is essential for the correct axonal development of MBs in Drosophila, suggests that the last common ancestor of protostomes might have possessed an ancient MB-like structure in the nervous system, and the MBs found in present animals may have evolved into specific neuropil structures in insect and lophotrochozoan brains supported in 
part by the retention of PsGEF in their genomes during evolution. Nematodes and crustaceans lost the full length PsGEF, and MB-like structures have disappeared as a result (Figure 9). However, the functions of PsGEF do not appear to be limited to MBs because it is also expressed in the optic and antennal lobes of adult Drosophila brain. Although MB-like structures are found in mollusks such as Achatina [38] and Octopus [39], it is not known whether limpets also have MB-like structures in their brains. It is thus possible that the conservation of PsGEF could be for other functional and developmental constraints.

Recent studies have demonstrated that the expression profile and roles of genes patterning the nervous system in the embryos of vertebrates and annelids are quite similar [9]. Since it is unlikely that this remarkable similarity arose from convergent evolution, they suggest that Urbilateria may have already had a quite complex CNS, which is also supported by comparative genomics [3,10]. It is therefore not surprising that the origin of MBs could be traced back to the last common ancestor of protostomes, in which ancient MB-like structures might have played a role in multimodal sensory integration and even learning and memory. To prove a single origin of MBs, it will be necessary to demonstrate that the same gene sets (for example, ey, toy, and dac) act similarly for the development of MBs in insects and lophotrochozoans. The homologs of these genes are also present in the vertebrate genomes, and they function in the early development of nervous systems. As these genes encode transcription factors, they could function as a common 'genetic code' to specify the brain region, giving rise to MBs and its vertebrate equivalents. Then, as a next level, a different set of genes such as PsGEF is necessary to generate the specific structures of MBs, which are present in the brains of insects and lophotrochozoans but not vertebrates. In this regard, $P S G E F$ is one of genes involved in generating the diversity of metazoan nervous systems.

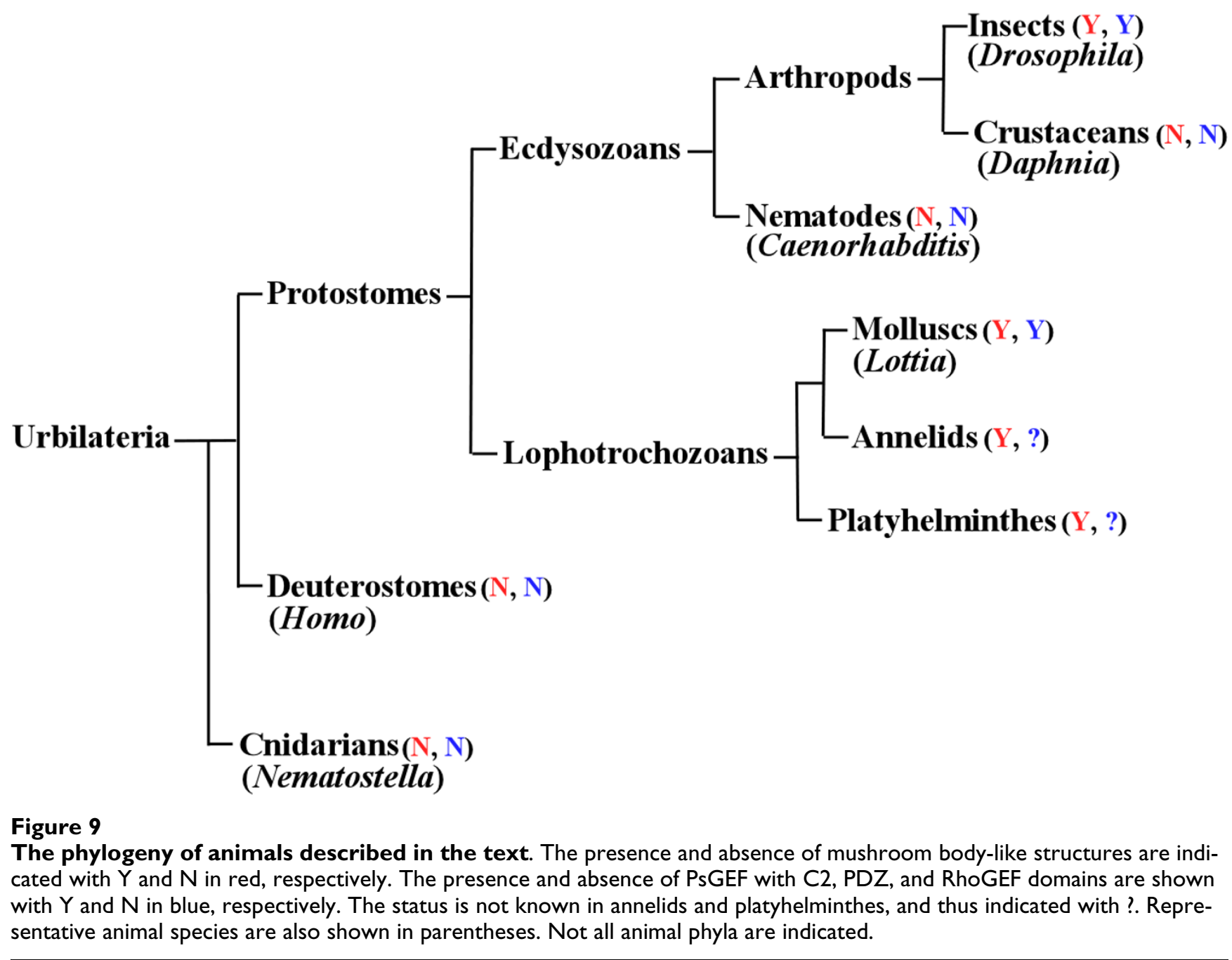




\section{Conclusion}

The specific acquisition of PsGEF by the last common ancestor of protostomes followed by the retention or loss in specific animal species during evolution demonstrates that there are some structural and/or functional features common between insect and lophotrochozoan nervous systems (for example, MBs), which are absent in all deuterostomes and cnidarians. PsGEF is therefore one of genes associated with the diversity of metazoan nervous systems.

\section{Methods}

Screening of insect-specific genes highly expressed in the Drosophila central nervous system

The identification of insect-specific orthologous genes has been previously described [1]. Drosophila genes were analyzed using FlyBase [40] in order to identify those in which GAL4 was inserted in their putative promoter regions. These GAL4 enhancer trap lines were individually crossed with UAS- $m C D 8:: G F P$; this was then followed by examination of green fluorescence protein (GFP) expression patterns in their progenies. We identified one line, namely, NP5114, which exhibits strong GFP expression in the embryonic and adult Drosophila CNS (Figure 4). In this line, GAL4 was inserted at a position 5' upstream of the first exon of DmPsGEF (CG14045) on the X chromosome (Figure 5). Other GAL4 lines in which GAL4 was inserted close to NP5114 (NP0264, NP1088, NP7169, NP3316, NP7265, NP3612, and NP3237) exhibit the same expression patterns, with variable intensities.

\section{Identification of alternatively spliced DmPsGEF mRNAs}

There is one DmPsGEF cDNA sequence deposited in an NCBI database (RE74757). This sequence corresponds to the short mRNA encoding DmPsGEF with the C2 and PDZ domains; this was confirmed by RT-PCR and rapid amplification of cDNA ends (3' RACE). To verify the presence of long mRNAs encoding DmPsGEF with the C2, PDZ, and RhoGEF domains, the corresponding fulllength cDNA was isolated by RT-PCR and then sequenced. The obtained sequence is identical to the one predicted from the genomic sequence (CG14045), except that a 108bp sequence is absent.

\section{Bioinformatics}

The amino acid sequences of TcPsGEF (GLEAN_01044), AmPsGEF (GB16089-PA), PhcPsGEF (PHUM010260PA), and LgPsGEF (fgenesh2_pg.C_sca_68000085) were retrieved from Baylor [41], BeeBase [42], VectorBase [43], and JGI [44], respectively. The functional domains of each protein were analyzed by NCBI CD search, and the sequence alignment of five PsGEF proteins was done by MEGA4 [45]. The genomic sequences of five PSGEF were also retrieved from above databases and FlyBase.

\section{Genetics}

$D m P s G E F$ deletion mutants were generated by the imprecise excision of NP5114. Approximately $300 w$ female revertants balanced with FM7 were individually crossed with FM7 males to establish the lines. The $B^{+}$adult males were collected from each line, and their genomic DNA was analyzed by PCR with the following two primers, namely, 5'-CACGGGATCTGCAGTGCAGACAACTCTT-3' and 5'-CAATCGCAGCTGTCAGTTCGGGAGGTGC-3', to identify the deletion mutants. The genomic PCR yielded a 7.5-kb band from the wild-type; thus, lines yielding bands smaller than $7.5 \mathrm{~kb}$ were analyzed further. We identified two large deletion mutants, namely, dmPsGEF ${ }^{\Delta 55}$ and $d m P s G E F \Delta 21$, and their breakpoints were determined by sequencing.

In order to visualize the alpha/beta lobes of MBs by GFP, $y, w, d m P s G E F^{\triangle 21} ; 201 Y-G A L 4$ females were crossed with UAS-mCD8::GFP males, and the resulting males were examined. To analyze the genetic interaction of DmPsGEF with Rac1, Rac2, Mtl, Rho1, and Pak, the $y, w$, $d m P s G E F^{\Delta 21}$ females were crossed with $y, w ; \operatorname{rac1} 1^{J 11} / C \gamma O, y$, $w ; \mathrm{rac}^{\Delta}, y, w ; \mathrm{mtl}^{\Delta} / \mathrm{TM} 3, y, w ; \mathrm{rho1}^{\mathrm{E3} .10} / \mathrm{C \gamma O}$, and $y, w ; p a k^{6} /$ TM3 males. The resulting males were then examined. To analyze the interaction of DmPsGEF with Cdc42, we crossed FM7/y, w, dmPsGEF $\triangle 21, c d c 42^{4}$ females with $y, w$, $d m P s G E F^{\Delta 21}$ males, and then the resulting $B^{+}$females were analyzed. More than 30 animals were analyzed for each case.

Single-cell MARCM clones (alpha/beta neurons) were generated by heat-shocking late-stage pupae of $y, w$, dmPsGEF $\triangle 21$, FRT19A/w, hs-FLP, tub-GAL80, FRT19A; UAS$m C D 8:: G F P /+; O K 107 /+$ at $37^{\circ} \mathrm{C}$ for $45 \mathrm{~min}$ [25]. As the wild-type control, $y, w$, FRT19A chromosomes were used.

\section{Immunohistochemistry}

The embryos were immunostained with rabbit anti-GFP antibodies (1,000-fold dilution) and horseradish peroxidase-conjugated anti-rabbit IgG antibodies (300-fold dilution) by using 3,3'-diaminobenzidine and nickel chloride as previously described [46]. The adult brains were dissected and fixed with 4\% paraformaldehyde/PBS on ice for $3 \mathrm{~h}$, permeabilized with phosphate-buffered saline (PBS) containing $0.5 \%$ TX-100 for $5 \mathrm{~min}$, and then blocked with PBS containing 5\% normal goat serum for $30 \mathrm{~min}$. They were then immunostained with 1D4 (4-fold dilution), rabbit anti-GFP antibody (1,000-fold dilution), Rhodamine anti-mouse IgG (300-fold dilution), and FITC anti-rabbit IgG (300-fold dilution).

\section{Reverse transcriptase-polymerase chain reaction}

Total RNA was isolated from NP5114,dmPsGEF ${ }^{\Delta 55}$, and $d m P s G E F^{\Delta 21}$ embryos. Thereafter, cDNAs were synthesized by using a reverse transcriptase (ReverTra Ace, TOYOBO). 
PCR was carried out using the following primers: 5'ATGACACGGATGCATCGCCACTCCAGTT-3' and 5'-TTAGACGAAGACACCTTTGCCTACCTCC-3' (for DmPsGEF short mRNA), 5'-ACCTTCAGCAAGGAGTCGATTGTGCCTG-3' and 5'-CTGCAGTTCGTTGATAACCGTGCTAAAG-3' (for DmPsGEF long mRNA), and 5'TGAGCATGAGCGCCACCTCGGATATCTA-3' and 5'-TGAGACTGGCGGATCTAGATGACGTAGT-3' (for CG14047 mRNA). The resulting PCR products were sequenced to verify their identities.

Total RNA was isolated from frozen samples of Daphnia pulex, and the cDNAs were then synthesized as mentioned above. PCR was carried out using the following primers: 5'-ATCGGCTCGCTACCTGAAATCCAACAGC-3' and 5'GTGACGCTTCCGCCTCCTGACGGTTTCT-3' (for SNAP_00018439 mRNA), 5'-ATGGCGGCCTCGTCTTCGGACCTTCAGG-3' and 5'-TCAAGTATCCTCGCAGCGTTCACCGAGT-3' (for SNAP_00018441 mRNA), and 5'GGTATCATGTCCGTGCAGCTGCACAAGT-3' and 5'CATCCATTTGACGGCGGATAAGGTCGAC-3' (for SNAP_00018442 mRNA). The resulting PCR products were sequenced to verify their identities.

\section{Ectopic expression of RhoGEF domain of DmPsGEF in HeLa cells}

A DNA fragment encoding the RhoGEF domain of DmPsGEF was PCR-amplified using DmPsGEF long CDNA as a template and the following primers: 5'-TTTTTGCGGCCGCATGAGCCGGCCGGCTACCGCATGCTCGG-3' and 5'TTTTTTAAGCTTACCAATATCACTCAGCGGCACCAG-

GGTG-3'. The resulting PCR product was digested with NotI and HindIII and inserted at the sites of the pCMVTag5A vector (Stratagene) that were treated with the same restriction enzymes. The resulting expression construct was introduced into HeLa cells with Effectene transfection reagent (Qiagen). Two days after transfection, the cells were fixed with $4 \%$ paraformaldehyde/PBS at room temperature for $15 \mathrm{~min}$; this was followed by permeabilization and blocking as done before. The transfected cells were detected by using rabbit anti-myc antibody $(1,000-$ fold dilution) and Rhodamine anti-rabbit IgG (300-fold dilution), and the F-actin was stained using FITC-phalloidin.

\section{Authors' contributions}

$\mathrm{NH}$ and KK performed all experiments described here. TK designed the experiments and wrote the manuscript. All authors have read and approved the final manuscript.

\section{Additional material}

\section{Additional file 1}

Supplementary material. The full length amino acid sequences of short and long DmPsGEF, TcPsGEF, PhcPsGEF, AmPsGEF, and LgPsGEF proteins are shown by a FASTA format.

Click here for file

[http://www.biomedcentral.com/content/supplementary/1741-

7007-7-21-S1.pdf]

\section{Additional file 2}

Figure S1. Alignment of amino acid sequences containing the C2, PDZ, and RhoGEF domains of five PsGEF proteins. The amino acid sequences containing the C2, PDZ, and RhoGEF domains of DmPsGEF (amino acid 272 to 1132), TcPsGEF (amino acid 601 to 1313), PhcPsGEF (amino acid 14 to 700), AmPsGEF (amino acid 595 to 1238), and LgPsGEF (amino acid 18 to 730 ) proteins are aligned by CLUSTALW program. The amino acid sequences of $C 2, P D Z$, and RhoGEF domains are highlighted by red, green, and blue, respectively. Identical amino acids are indicated by asterisks, and the conserved amino acids are shown by either dots or colons. Only C2, PDZ, and RhoGEF domains show the significant similarity between five PsGEF proteins.

Click here for file

[http://www.biomedcentral.com/content/supplementary/17417007-7-21-S2.pdf]

\section{Acknowledgements}

We thank H Hing, RG Fehon, K Furukubo-Tokunaga, the Kyoto Drosophila Genetic Resource Center, and Bloomington Stock Center for the fly stocks. We are grateful to $S$ Tochinai for providing us Daphnia pulex, and $M$ Mizunami for discussion. We also thank $\mathrm{C}$ Hama and the Developmental Studies Hybridoma Bank for providing us the anti-Trio and monoclonal antibody ID4, respectively. This study was supported by a Grant-in-Aid for Scientific Research from the Japan Society for the Promotion of Science to TK.

\section{References}

I. Wyder S, Kriventseva EV, Schröder R, Kadowaki T, Zdobnov EM: Quantification of ortholog losses in insects and vertebrates. Genome Biol 2007, 8:R242.

2. Raible F, Tessmar-Raible K, Osoegawa K, Wincker P, Jubin C, Balavoine G, Ferrier D, Benes V, de Jong P, Weissenbach J, Bork P, Arendt $D$ : Vertebrate-type intron-rich genes in the marine annelid Platynereis dumerilii. Science 2005, 3 1 0: I 325-1 326.

3. Moroz LL, Edwards JR, Puthanveettil SV, Kohn AB, Ha T, Heyland A, Knudsen B, Sahni A, Yu F, Liu L, Jezzini S, Lovell P, lannucculli W, Chen M, Nguyen T, Sheng H, Shaw R, Kalachikov S, Panchin YV, Farmerie W, Russo JJ, Ju J, Kandel ER: Neuronal transcriptome of Aplysia: Neuronal compartments and circuitry. Cell 2006, I 27:1453-1467.

4. De Robertis EM: Evo-Devo: Variations on ancestral themes. Cell 2008, I32:185-195.

5. Lowe CJ, Wu M, Salic A, Evans L, Lander E, Stange-Thomann N, Gruber CE, Gerhart J, Kirschner M: Anteroposterior patterning in hemichordates and the origins of the chordate nervous system. Cell 2003, I I 3:853-865.

6. Lowe CJ, Terasaki M, Wu M, Freeman RM Jr, Runft L, Kwan K, Haigo S, Aronowicz J, Lander E, Gruber C, Smith M, Kirschner M, Gerhart J: Dorsoventral patterning in hemichordates: insights into early chordate evolution. PLOS Biol 2006, 4:e29I.

7. Holland ND: Early central nervous system evolution: an era of skin brains? Nat Rev Neurosci 2003, 4:617-627. 
8. Lichtneckert R, Reichert $\mathrm{H}$ : Insights into the urbilaterian brain: conserved genetic patterning mechanisms in insect and vertebrate brain development. Heredity 2005, 94:465-477.

9. Denes AS, Jékely G, Steinmetz PR, Raible F, Snyman H, Prud'homme B, Ferrier DE, Balavoine G, Arendt D: Molecular architecture of annelid nerve cord supports common origin of nervous system centralization in bilateria. Cell 2007, 129:277-288.

10. Mineta K, Nakazawa M, Cebria F, Ikeo K, Agata K, Gojobori T: Origin and evolutionary process of the CNS elucidated by comparative genomics analysis of planarian ESTs. Proc Natl Acad Sci USA 2003, 100:7666-7671.

II. Strausfeld NJ, Hansen L, Li Y, Gomez RS, Ito K: Evolution, discovery, and interpretations of arthropod mushroom bodies. Learn Mem 1998, 5: I I-37.

12. Fahrbach SE: Structure of the mushroom bodies of the insect brain. Annu Rev Entomol 2006, 5 I:209-232.

13. Noveen A, Daniel A, Hartenstein V: Early development of the Drosophila mushroom body: the roles of eyeless and dachshund. Development 2000, I 27:3475-3488.

14. Martini SR, Roman G, Meuser S, Mardon G, Davis RL: The retinal determination gene, dachshund, is required for mushroom body cell differentiation. Development 2000, 127:2663-2672.

15. Kurusu M, Nagao T, Walldorf U, Flister S, Gehring WJ, FurukuboTokunaga K: Genetic control of development of the mushroom bodies, the associative learning centers in the Drosophila brain, by the eyeless, twin of eyeless, and Dachshund genes. Proc Natl Acad Sci USA 2000, 97:2 I40-2। 44.

16. Osumi N, Shinohara H, Numayama-Tsuruta K, Maekawa M: Concise review: Pax6 transcription factor contributes to both embryonic and adult neurogenesis as a multifunctional regulator. Stem Cells 2008, 26:1663-1672.

17. Sutton RB, Davletov BA, Berghuis AM, Südhof TC, Sprang SR: Structure of the first $\mathrm{C2}$ domain of synaptotagmin I: a novel $\mathrm{Ca2}+$ phospholipid-binding fold. Cell 1995, 80:929-938.

18. Davletov BA, Südhof TC: $\mathbf{C a}(2+)$-dependent conformational change in synaptotagmin I. J Biol Chem I994, 269:28547-28550.

19. Ponting CP, Phillips C, Davies KE, Blake DJ: PDZ domains: targeting signalling molecules to sub-membranous sites. Bioessays 1997, 19:469-479.

20. Kim E, Sheng M: PDZ domain proteins of synapses. Nat Rev Neurosci 2004, 5:77I-78I.

21. Zheng Y: Dbl family guanine nucleotide exchange factors. Trends Biochem Sci 200I, 26:724-732.

22. Shi CS, Lee SB, Sinnarajah S, Dessauer CW, Rhee SG, Kehrl JH: Regulator of G-protein signaling 3 (RGS3) inhibits Gbetalgamma 2-induced inositol phosphate production, mitogen-activated protein kinase activation, and Akt activation. I Biol Chem 200I, 276:24293-24300.

23. Joint Genome Institute (JGI) database [http://genome.jgipsf.org/Dappul/Dappul.home.html]

24. Awasaki T, Saito M, Sone M, Suzuki E, Sakai R, Ito K, Hama C: The Drosophila trio plays an essential role in patterning of axons by regulating their directional extension. Neuron 2000 26:||9-|3|.

25. Lee T, Lee A, Luo L: Development of the Drosophila mushroom bodies: sequential generation of three distinct types of neurons from a neuroblast. Development 1999, 1 26:4065-4076.

26. Ng J, Nardine T, Harms M, Tzu J, Goldstein A, Sun Y, Dietzl G, Dickson B, Luo L: Rac GTPases control axon growth, guidance and branching. Nature 2002, 416:442-447.

27. Ridley AJ, Paterson HF, Johnston CL, Diekmann D, Hall A: The smal GTP-binding protein rac regulates growth factor-induced membrane ruffling. Cell 1992, 70:40I-410.

28. Nobes $C D$, Hall $A:$ Rho, rac, and cdc42 GTPases regulate the assembly of multimolecular focal complexes associated with actin stress fibers, lamellipodia, and filopodia. Cell 1995, 8I:53-62.

29. Tissot M, Stocker RF: Metamorphosis in drosophila and other insects: the fate of neurons throughout the stages. Prog Neurobiol 2000, 62:89-1II

30. Porcelli D, Barsanti P, Pesole G, Caggese C: The nuclear OXPHOS genes in insecta: a common evolutionary origin, a common cis-regulatory motif, a common destiny for gene duplicates. BMC Evol Biol 2007, 7:215.

31. Patthy L: Modular assembly of genes and the evolution of new functions. Genetica 2003, I | 8:217-231.
32. Mushegian AR, Garey JR, Martin J, Liu LX: Large-scale taxonomic profiling of eukaryotic model organisms: A comparison of orthologous proteins encoded by the human, fly, nematode, and yeast genomes. Genome Res 1998, 8:590-598.

33. Giribet G, Edgecombe GD, Wheeler WC: Arthropod phylogeny based on eight molecular loci and morphology. Nature 200I, 4I3:|57-|6I.

34. Hwang UW, Friedrich M, Tautz D, Park CJ, Kim W: Mitochondrial protein phylogeny joins myriapods with chelicerates. Nature 200I, 4I 3: I54-157.

35. Strausfeld NJ, Strausfeld CM, Stowe S, Rowell D, Loesel R: The organization and evolutionary implications of neuropils and their neurons in the brain of the onychophoran Euperipatoides rowelli. Arthropod Struct Dev 2006, 35:169-196.

36. $\mathrm{Ng}$ J, Luo L: Rho GTPase regulates axon growth through convergent and divergent signaling pathway. Neuron 2004, 44:779-793.

37. Sone M, Suzuki E, Hoshino M, Hou D, Kuromi H, Fukata M, Kuroda S, Kaibuchi K, Nabeshima Y, Hama C: Synaptic development is controlled in the periactive zones of Drosophila synapses. Development 2000, 127:4157-4168.

38. Chase $R$, Tolloczko $B$ : Tracing neural pathways in snail olfaction: from the tip of the tentacles to the brain and beyond. Microsco Res Tech 1993, 24:21 4-230.

39. Young JZ: The Anatomy of the Nervous System of Octopus vulgaris Oxford: Clarendon Press; 1971.

40. FlyBase [http://flybase.org/]

41. Baylor Tribolium castaneum Genome Project database [http:/ /www.hgsc.bcm.tmc.edu/projects/tribolium/]

42. BeeBase Hymenoptera Genome Database [http://www.bee base.org/]

43. VectorBase Database [http://phumanus.vectorbase.org/ index.php]

44. Joint Genome Institute [http://genome.jgi-psf.org/Lotgil/ Lotgil.home.html]

45. Tamura K, Dudley J, Nei M, Kumar S: MEGA4: Molecular Evolutionary Genetics Analysis (MEGA) software version 4.0. Mol Biol Evol 2007, 24:1596-1599.

46. Funada $M$, Hara $\mathrm{H}$, Sasagawa $\mathrm{H}$, Kitagawa $\mathrm{Y}$, Kadowaki $\mathrm{T}$ : A honey bee Dscam family member, AbsCAM, is a brain-specific cell adhesion molecule with the neurite outgrowth activity which influences neuronal wiring during development. Eur J Neurosci 2007, 25:168-180.
Publish with Bio Med Central and every scientist can read your work free of charge

"BioMed Central will be the most significant development for disseminating the results of biomedical research in our lifetime. "

Sir Paul Nurse, Cancer Research UK

Your research papers will be:

- available free of charge to the entire biomedical community

- peer reviewed and published immediately upon acceptance

- cited in PubMed and archived on PubMed Central

- yours - you keep the copyright

Submit your manuscript here:

http://www.biomedcentral.com/info/publishing_adv.asp
BiolMedcentral 